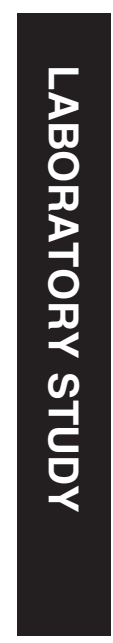

\section{Vital dyes increase the rigidity of the internal limiting membrane}

C Haritoglou', S Mauell2, M Benoit², RG Schumann ${ }^{1}$, PB Henrich ${ }^{3}$, A Wolf ${ }^{1}$ and A Kampik ${ }^{1}$
${ }^{1}$ Department of Ophthalmology, LudwigMaximilians-University, Munich, Germany

${ }^{2}$ Department of Applied Physics and Center for NanoScience, LudwigMaximilians-University, Munich, Germany

${ }^{3}$ Department of Ophthalmology, University Hospital Basel, Basel, Switzerland

Correspondence: C Haritoglou, Department of Ophthalmology, Pathology and Vitreoretinal Unit, Ludwig-MaximiliansUniversity, Mathildenstr. 8, München, Bavaria 80336, Germany.

Tel: +49 8951603811 ; Fax: +49895160 4778. E-mail: christos.haritoglou@ med.uni-muenchen.de

Received: 7 May 2013 Accepted: 15 July 2013 Published online: 16 August 2013

\begin{abstract}
Purpose To assess the stiffness of the natural human internal limiting membrane (ILM) and evaluate potential changes of the mechanical properties following staining with brilliant blue (BB) and indocyanine green (ICG).

Methods Unstained ILM specimens were obtained during ophthalmic surgical procedures. After removal, the specimens were dissected into five parts. Two fragments were stained with BB and ICG, respectively, for $1 \mathrm{~min}$, another two specimens were stained similarly followed by additional subsequent illumination using a standard light source (PENTA LUX x 50, Ophthalmologische Systeme GmbH Fritz Ruck). The fifth part served as an untreated control. All specimens were then analyzed using atomic force microscopy (AFM) in contact mode with a scan rate of $0.6 \mathrm{~Hz}$. Two scan regions of $10 \times 10 \mu \mathrm{m}$ were chosen and stiffness was determined by using AFM in a force spectroscopy mode. The force curves were plotted with a data rate of $5000 \mathrm{~Hz}$. In all specimens both the retinal side and vitreal side were analyzed.

Results Staining resulted in a significant increase in tissue stiffness. An increase was seen both for the vitreal (BB: $P<0.001$; ICG: $P<0.01$ ) and retinal side (BB: $P<0.01$; ICG: $P<0.01$ ), with the retinal side being significantly stiffer in all control and stained samples. Additional illumination after staining did further increase tissue rigidity in most samples but not significantly.

Conclusions Staining significantly increases the stiffness of the human ILM. This might explain the fact that the stained ILM can be removed more easily and in larger fragments during vitreoretinal surgical procedures compared with unstained ILM.
\end{abstract}

Eye (2013) 27, 1308-1315; doi:10.1038/eye.2013.178; published online 16 August 2013

Keywords: brilliant blue; indocyanine green; stiffness; internal limiting membrane; AFM

Introduction

It is widely accepted among vitreoretinal surgeons that removal of the internal limiting membrane (ILM) during surgery for tractional maculopathies improves both functional and anatomical success ${ }^{1-4}$ and may help to decrease the incidence of recurrences of the disease. ${ }^{4}$ Peeling of the ILM is a challenging procedure and requires surgical skills and experience. In order to facilitate visualization of this transparent structure and to reduce excessive and prolonged manipulation on the retina, staining substances have been introduced over the last decade. The first dye suggested was the cyanine dye indocyanine green (ICG) ${ }^{5}$ in the year 2000. ICG became a subject of debate because of its potential toxicity, most likely related to its chemical instability and a photosensitizing effect that has been demonstrated experimentally, ${ }^{6-9}$ which may lead to functional deficits in clinical practice. ${ }^{10,11}$ A few years later, the triarylmethane dye brilliant blue became commercially available. On the basis of clinical and experimental studies published so far, brilliant blue seems to provide an improved safety profile compared with ICG. ${ }^{12-16}$ Very recently a comparative study evaluating the microstructure of the inner and outer retina and visual function after macular hole surgery using brilliant blue or indocyanine green revealed that postoperative visual acuity and retinal sensitivity were better in eyes after BBG-assisted vitrectomy. ${ }^{17}$

However, regardless which dyes the surgeon may choose for the surgical intervention, it has 
been reported that the stained ILM provides an increased rigidity and can be peeled off more easily and in larger fragments as compared with the unstained tissue. ${ }^{15}$

Atomic Force Microscopy (AFM) is a well established examination technique that has been used to measure the thickness and rigidity of human ocular basement membranes such as the unstained ILM. ${ }^{18,19}$ The aim of the present investigation was to assess and quantify in vitro changes of the ILM of the human retina after staining with either brilliant blue or ICG with and without subsequent illumination using a standard surgical light (PENTA LUX x 50, Ophthalmologische Systeme GmbH Fritz Ruck, Eschweiler, Germany) source compared with unstained ILM using AFM.

\section{Methods}

The study was approved by the local Institutional Review Board.

\section{Tissue preparation and handling}

Specimens of unstained human ILM were obtained during vitreoretinal procedures to treat macular holes and epiretinal membranes. Vitreoretinal surgery consisted of a standard 23-gauge vitrectomy, induction of a posterior vitreous detachment, and removal of epiretinal membranes if present using a pair of end-gripping 23-gauge forceps. This was followed by a peeling of the unstained ILM (surgeon AK), which was immediately placed in BSS and handed over to a technician (RS) for further processing.

Immediately after removal from the eye, the ILM specimen was placed on a glass slide and divided into five parts. Each piece was placed inside of a BSS droplet on a microscope slide (SuperFrost Plus, Gerhard Menzel $\mathrm{GmbH}$, Braunschweig, Germany) that had been coated with poly-L-lysine. BSS was removed slowly while the membrane was carefully smoothed on the glass using a glass needle avoiding dehydration of the specimen during preparation. A circle was then drawn around each ILM fragment with a PAP pen. Then, a drop of brilliant blue $0.025 \%$ (Brilliant Peel; Fluoron $\mathrm{GmbH}$, NeuUlm, Germany) or indocyanine green $0.05 \%$ was placed on two fragments each. ICG dye was prepared as follows: $25 \mathrm{mg}$ of dry ICG substance (Pulsion, Munich, Germany) was first dissolved in $5 \mathrm{ml}$ sterile water as recommended by the manufacturer. One microliter of this $0.5 \%$ solution was then diluted with $9 \mathrm{ml}$ BSS plus resulting in a final concentration of $0.05 \%$. Excessive dye was then rinsed off after $1 \mathrm{~min}$. One of these specimens was then illuminated using a standard light source used for vitreoretinal surgery (PENTA LUX x 50,

Ophthalmologische Systeme GmbH Fritz Ruck) for one additional minute. The remaining unstained fragment served as a control. Therefore, atomic force microscopy could be performed in five fragments of each specimen: an untreated and unstained control, one after staining with brilliant blue, one after staining with brilliant blue and subsequent illumination, one after staining with ICG, and one after staining with ICG and subsequent illumination. All in all, 20 ILM specimens were analyzed for each dye, with the retinal side (BBG $[n=8]$; ICG $(n=11))$ and the vitreal side (BBG $(n=12)$; ICG $(n=9))$ being evaluated separately.

ILM specimens were also stained for laminin after AFM measurements in order to determine the orientation (vitreal or retinal side) on the glass slide. For this purpose, the ILM samples were first washed three times with 0.01 molar phosphate buffered saline (PBS). In a second step, a droplet of $120 \mu \mathrm{l} 4 \%$ paraformaldehyde was placed onto the membrane for about $15 \mathrm{~min}$. After another washing step, $120 \mu \mathrm{l}$ laminin labeling antibody 1:100 (Polyclonal Rabbit Anti-Laminin, Dako Deutschland GmbH, Hamburg, Germany) was added, and the sample was stored in a humidity box at $8^{\circ} \mathrm{C}$ for $24 \mathrm{~h}$. Thereafter, a third washing step was performed followed by the staining with $100 \mu \mathrm{l}$ cy3 fluorescent dye-coupled secondary antibody 1:100 (Donkey anti-Rabbit IgG $(\mathrm{H}+\mathrm{L})-\mathrm{Cy} 3$, Dianova $\mathrm{GmbH}$, Hamburg, Germany) that was applied for $45 \mathrm{~min}$. All samples were rinsed again in PBS, and $20 \mu \mathrm{l}$ of mounting medium (Fluorescence Mounting Medium, Dako Deutschland $\mathrm{GmbH}$ ) was applied onto the ILM, and then covered with a round cover slip and allowed to solidify at room temperature for about $3 \mathrm{~h}$.

All ILM specimens were also evaluated using a fluorescence microscope. All fluorescence imaging experiments were performed using an Axiovert 25 inversion fluorescence microscope (Carl Zeiss AG; Oberkochen, Germany). As a light source, a mercury vapor lamp (HBO 50; Osram, Munich, Germany) was used. Using the excitation filter ET545/25x (Chroma; Taoyuan Hsien, Taiwan), the $546 \mathrm{~nm}$ peak in the mercury spectrum was selected. The light was reflected by the dichroic T565LP (Chroma) through the objective (Fluar 10x/0.50; Carl Zeiss AG) onto the sample and excited the cy3 dye used for labeling. The emitted light passed the dichroic, passed the emission filter ET605/70 m (Chroma) and was detected using a CCD camera (Infinity 2; BFI Optilas; Dietzenbach, Germany) and processed using a computer. The camera was controlled by the software 'Infinity Capture 5.0.0' (Lumenera, Leipzig, Germany).

\section{Atomic force microscopy measurements}

All AFM imaging and force indentation experiments were performed using a Nanowizard II Atomic Force 
Microscope (JPK Instruments, Berlin, Germany), which was mounted on an inverted microscope. For imaging and stiffness measurements with Nanowizard II AFM, standard silicon nitride triangular cantilevers (MLCT, Veeco Instruments, Plainview, NY, USA) with integrated sharp silicon-nitride pyramidal tips (nominal spring constant $k=0.1 \mathrm{~N} / \mathrm{m}$, apex angle $\alpha \sim 35^{\circ}$ ) were used. Before every measurement, the spring constant value was determined using the thermal fluctuation method. The samples were imaged in contact mode with a scan rate of $0.6 \mathrm{~Hz}$ while kept in PBS at room temperature. Two scan regions of $10 \times 10 \mu \mathrm{m}$ were chosen on the sample. Stiffness was determined using AFM in a force spectroscopy mode. Briefly, indentations were made over an $8 \times 8$ point grid at a rate of one load/unload cycle per $2.4 \mathrm{~s}$ with a maximal load of $5 \mathrm{nN}$. The force curves were plotted with an approaching speed of $3 \mu \mathrm{m} / \mathrm{s}$, a retraction speed of $7 \mu \mathrm{m} / \mathrm{s}$, and a data rate of $5000 \mathrm{~Hz}$. Assessment of the dynamic indentation modulus $\mathrm{E}$ of each force-indentation curve was plotted off-line using a data processing software (JPK Instruments AG).

Therefore, the height data of the scan regions were calculated to tip-sample separation and plotted against indentation force. After fitting the Sneddon model ${ }^{19}$ to the data, the resulting elasticity modulus was averaged for each scan region.

The stiffness of an elastic material is quantified by Young's modulus (tensile modulus, elastic modulus, modulus of elasticity), a material property used to characterize materials. The SI unit of Young's modulus is the pascal $\left(\mathrm{Pa}\right.$ or $\left.\mathrm{Nm}^{-2}\right)$.

\section{Identification of ILM sample orientation}

During the measurements it became necessary to determine the orientation of the sample on the glass slide. Preliminary measurements on ILM specimens stained during vitreoretinal surgery within the human eye and removed for analysis confirmed that there is a difference in elasticity on the retinal and on the vitreal side of the ILM as described in the literature. ${ }^{20,21}$ As a consequence, a reliable orientation of the ILM specimens for each measurement is crucial in order to obtain reliable results. In our study, AFM imaging was used to clearly identify the rough retinal surface and the smooth vitreal surface as topographic landmarks (Figure 1a).

Additional tests for correct tissue orientation were performed using a protocol for staining for laminin and consequent fluorescence microscopy (Figure 1b). This was performed after the AFM measurements had been obtained to exclude potential effects of the illumination by the microscope on the result. a
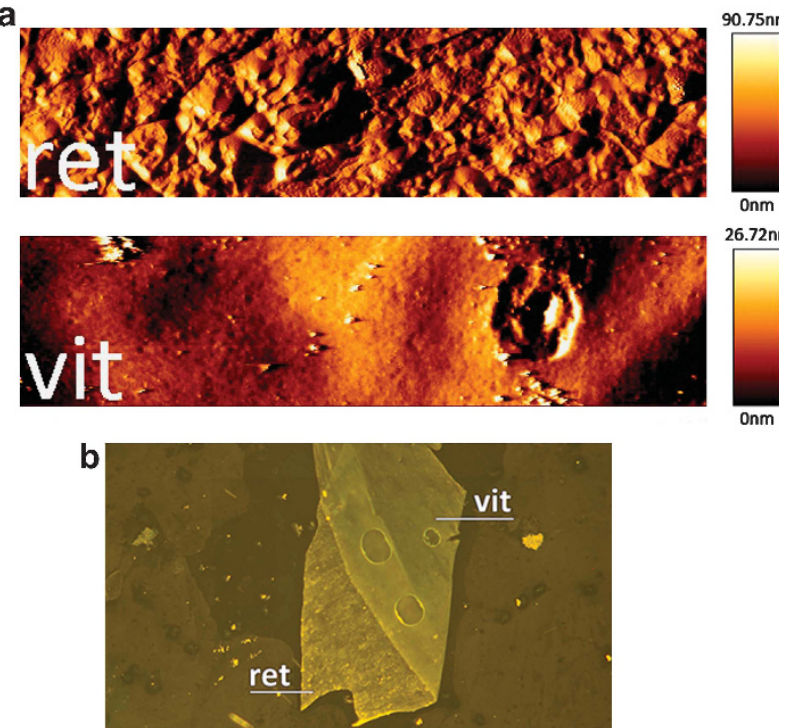

Figure 1 (a) AFM contact mode images with a size of $80 \times 20 \mu \mathrm{m}$ of both ILM sides. The smooth vitreal side can be clearly distinguished from the rough and undulated retinal side (roughness retinal: $9075 \mathrm{~nm}$, vitreal: $2672 \mathrm{~nm}$ ). The deflection signal is shown in units of $\mathrm{nm}$. (b) ILM stained for laminin and imaged using fluorescence microscopy (magnification: $\times 10$ ). The smooth vitreal and rough retinal side can be determined easily. The ILM sample is partially flipped over, and hence the difference of both sides can be appreciated.

\section{Results}

Staining of the ILM-samples resulted in a statistically significant increase in tissue rigidity compared with controls (Figure 2). Of note, the retinal surface of all specimens, both control and stained tissue, was about fivefold stiffer than the vitreal surface. Compared with unstained controls of ILM, the stiffness of the tissue significantly increased with the use of brilliant blue or ICG for staining. This was true both for the vitreal surface (BBG: $P<0.001$; ICG: $P<0.01$ ) as well as the retinal surface (BBG: $P<0.01$; ICG: $P<0.01$ ). The difference in stiffness comparing the retinal versus the vitreal surface seen in the controls remained unchanged after staining of the tissue and subsequent illumination. Calculated for each dye, there was a trend to a further increase in stiffness after illumination of the stained tissue, but this effect did not reach statistically significant levels (Figure 2, Table 1). Comparing the two dyes after illumination, there were statistical differences seen depending on which side was measured (BBL versus ICGL measured at the vitreal side, Table 1).

\section{Relative elasticity changes and the effect of Illumination}

Comparing the Young's moduli in relation to the corresponding control sample, we noted that except for ILM samples after brilliant blue staining and 


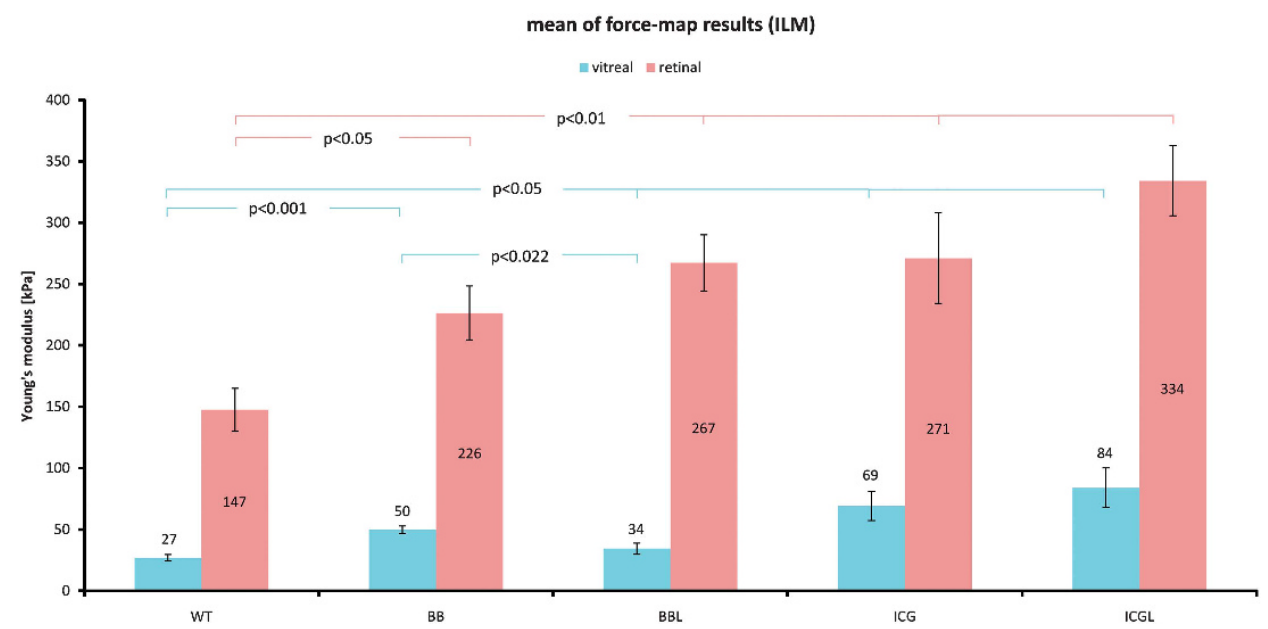

Figure 2 Averages of all map-results of ILM are plotted with respect to the measured side (vitreal: blue; retinal: red), with error bars showing the SE of the mean. Indicated $P$-values hold a significant level of $5 \%$ and are calculated from a $t$-test. BB, brilliant blue; BBL, brilliant blue and illumination; ICG, indocyanine green; ICGL, indocyanine green and illumination; WT, control. Differences between control (WT) and BBL and ICGL were also significant both for the retinal as well as the vitreal surface.

Table 1 Significant levels comparing dyes and controls with regard to the increase of stiffness measured at the retinal and vitreal surface of the ILM

\begin{tabular}{lllll}
\hline$P<$ & Control & $B B$ & $B B L$ & ICG \\
\hline ILM measurements retinal surface & & & & \\
$\quad$ BB & 0.05 & & & \\
BBL & 0.001 & NS & & \\
ICG & 0.01 & NS & NS & \\
ICGL & 0.001 & 0.05 & NS & NS \\
& & & & \\
ILM measurements vitreal surface & & & & \\
BB & 0.001 & & & \\
BBL & NS & 0.05 & & \\
ICG & 0.05 & NS & 0.01 & \\
ICGL & 0.01 & NS & 0.01 & NS
\end{tabular}

Abbreviations: $\mathrm{BB}$, brilliant blue; $\mathrm{BBL}$, brilliant blue and illumination; ICG, indocyanine green; ICGL, indocyanine green and illumination; NS, not significant.

illumination (BBL) the relative elasticity change of the vitreal side was more pronounced compared with the retinal side (Figure 3, Table 2). Of note, values for ICG with and without illumination were higher compared with brilliant blue in ILM specimens. In addition, except for $\mathrm{BB} / \mathrm{BBL}$, the relative elasticity of stained specimens increased by 1.2-fold following illumination (retinal surface: $1.18 \pm 0.02$ for BB/BBL, $1.23 \pm 0.04$ for ICG/ ICGL; vitreal side: $0.69 \pm 0.05$ for $\mathrm{BB} / \mathrm{BBL}$ and $1.21 \pm 0.01$ for ICG/ICGL). This implies an additional stiffening effect of the tissue following illumination, which was not statistically significant. The gradient was around 1.2 for all dyes and tissues (except for vitreal BBL) (Table 3).

\section{Discussion}

The ILM of the retina is a multilaminar structure mainly composed of type IV collagen, laminin, and fibronectin and essentially represents the basement membrane of Müller cells. Although the inner vitreal surface of the ILM appears smooth, the ILM has a rough, undulated outer retinal surface, a finding being most remarkable at the posterior pole. This morphological difference helps to identify the respective side of the ILM in histological specimens as performed in the present study and helps to correlate ILM samples with regard to their localization. The ILM is adherent to the type II collagen fibers of the vitreal cortex, ${ }^{22}$ helping vitreal tractional forces to be transmitted to the retinal surface, contributing to the pathogenesis of several tractive maculopathies such as macular pucker, macular hole, or vitreoretinal traction syndrome. Therefore, ILM removal is considered to be an important step to successfully treat these conditions and prevent recurrences in order to remove all tractional forces adherent to the ILM.

Of note, the ILM is a very delicate structure that varies in thickness, being thicker posteriorly $(0.5-3.2 \mu \mathrm{m})$ and thinner at the fovea and the disc $(0.01-0.10 \mu \mathrm{m}){ }^{23}$ The ILM is a surprisingly rigid structure. Previous AFM studies showed that the mechanical strength is in the $\mathrm{mPa}$ range, very similar to the articular cartilage and about 1000 -fold stronger than cell layers. ${ }^{18,24,25}$ Our results underline previous reports ${ }^{20}$ describing that the average stiffness of the ILM is over fivefold higher at the retinal side as compared with the vitreal side. This difference nicely explains the fact that the ILM curls up towards its vitreal side after peeling. The high 


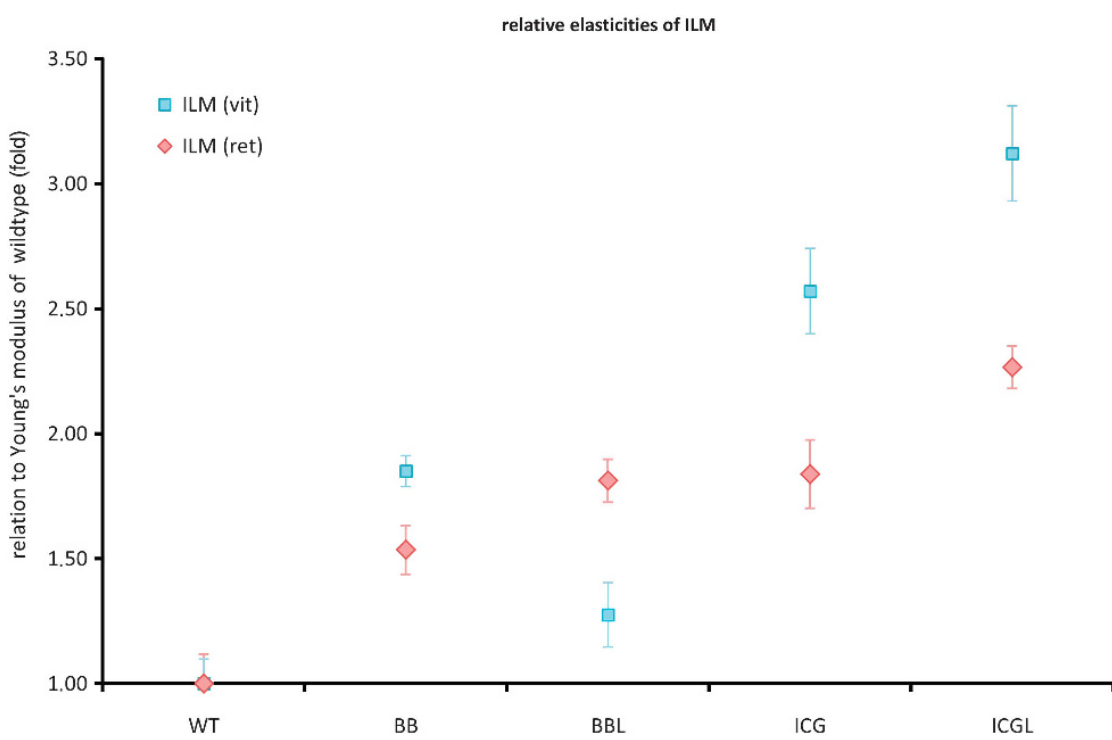

Figure 3 Relative elasticity changes of the ILM measured on the vitreal and retinal side of the tissue. BB, brilliant blue; BBL, brilliant blue and illumination; ICG, indocyanine green; ICGL, indocyanine green and illumination; WT, control.

Table 2 Relative elasticities: each Young's modulus is divided by its respective control value

\begin{tabular}{lccccr}
\hline & Control & $B B$ & $B B L$ & ICG & ICGL \\
\hline Retinal ILM & $1.00 \pm 0.12$ & $1.54 \pm 0.10$ & $1.81 \pm 0.09$ & $1.84 \pm 0.14$ & $2.27 \pm 0.09$ \\
Vitreal ILM & $1.00 \pm 0.10$ & $1.85 \pm 0.06$ & $1.27 \pm 0.13$ & $2.57 \pm 0.17$ & $3.12 \pm 0.19$ \\
\hline
\end{tabular}

Abbreviations: BB, brilliant blue; BBL, brilliant blue and illumination; ICG, indocyanine green; ICGL, indocyanine green and illumination. Except for BBL the values of vitreal ILM exceed those of retinal ILM.

mechanical strength of the natural, unstained ILM gives the possibility to grasp the ILM using an end-gripping forceps and to peel off mechanically the ILM from the underlying nerve fiber layer.

As unaided, unstained ILM peeling requires great surgical skills. Staining substances were introduced during the last years that greatly facilitate the visualization and removal of the ILM during vitreomacular surgeries. Both dyes in clinical use, ICG and $\mathrm{BBG}$, provide selective staining properties and a good enough contrast to identify and peel off the ILM. The advantage of ILM staining is obvious. Better visualization is supposed to result in an easier removal without damage to the underlying nerve fiber layer and consequently better functional results postoperatively.

However, it is a commonly reported intraoperative observation that stained ILM can be peeled of more easily and in larger fragments as compared with the unstained ILM. On the basis of the present study, we hypothesize that there are specific tissue dye interactions that increase the stiffness of ILM, and we conclude that the observed change in stiffness is a very likely explanation for the easier removal of ILM after staining. The effect appears more pronounced when using ICG as
Table 3 Gradients between the dye-specific lightexposed and unexposed samples

\begin{tabular}{lcc}
\hline & $B B L / B B$ & $I C G / I C G L$ \\
\hline ILM retinal & $1.18 \pm 0.02$ & $1.23 \pm 0.04$ \\
ILM vitreal & $0.69 \pm 0.05$ & $1.21 \pm 0.01$ \\
\hline
\end{tabular}

Abbreviations: $\mathrm{BB}$, brilliant blue; BBL, brilliant blue and illumination; ICG, indocyanine green; ICGL, indocyanine green and illumination. Except for the gradient of BBL and BB the gradient values are located around 1.2.

compared with BBG. Interestingly, illumination using a standard surgical light source did not contribute a significant effect to even further increase the stiffness of the tissue. However, except for brilliant blue, illumination increased the stiffness in all examined tissues and irrespective of the measured surface in a very consistent manner by 1.2-fold. Interestingly, the change of elasticity was more pronounced at the vitreal side compared with the retinal side. This may be explained by the presence of collagen remnants adherent to the vitreal side of the specimens that further increase the stiffening effect after staining using the dye. 
One may argue that the easier removal after staining is merely related to the better visualization of the ILM. Better visualization undoubtedly helps to identify the ILM and initiate the peeling at the correct plane of dissection, but does not explain the altered mechanical properties as such. Alternatively, one may suggest that the adhesion of the stained ILM is decreased, implying that there is an alteration of the cleavage plane or some kind of loosening of the ILM from the underlying nerve fiber layer as an effect of the dye applied. The issue of the alteration of the cleavage plane was extensively discussed in the literature as a possible effect of ICGrelated phototoxicity. ${ }^{10,26}$ Under the light of the present study and its results, this hypothesis must be challenged as BB and ICG reveal quite similar changes concerning their mechanical properties, and no phototoxicity is known for BB (see below).

However, the underlying mechanism for the observations described in the present study remains hypothetical. Looking at the published literature there are some studies describing an alteration of the mechanical properties of the ILM after staining: For ICG, Wollensak et al. ${ }^{27,28}$ reported an increased stiffness of the ICG-stained ILM following $3 \mathrm{~min}$ of illumination of the stained post-mortem porcine retina, showing a significant increase in ultimate force by $45 \%$ and a decrease in ultimate elongation by $24 \%$. Interestingly, no such effect was seen without subsequent illumination, suggesting a light-dependent process. They, therefore, concluded that the stiffening effect of ICG combined with light is related to a photosensitizing effect of ICG by the formation of a triplet state of the ICG molecule and reactive oxygen species (type I reaction of photooxidation). ${ }^{27,29}$ Reactive oxygen species then lead to photooxidative damage of cells and physical crosslinking of collagen fibers such as the type IV collagen of the ILM. ${ }^{27,28,30}$ However, in our own experiments using AFM in fresh human tissue, an increase in stiffness was seen also for ICG without subsequent illumination maybe because the specimens were not kept in darkness as described for Wollensak's experimental setting. ${ }^{27}$ It is widely accepted that the potential toxic effects of ICG $^{10,11,31,32}$ as described clinically are very likely related to photosensitizing properties of ICG as described above and the decomposition products of the ICG molecule after illumination. ${ }^{7,8,33}$

Importantly, other additional relevant tissue-dye interactions seem to have a role as a causative effect for the increase in tissue stiffness, as increased rigidity was also seen for BBG in our experiments. In contrast to ICG, BBG, a triarylmethane dye, has no known photosensitizing properties. This observation is of relevance as histological evaluations of ICG-stained ILM specimens obtained during macular hole surgery revealed the striking presence of retinal debris and large cellular fragments adherent to the retinal surface of the ILM. ${ }^{10}$ As such extensive cellular components had not been seen before the use of ICG in macular surgery, it was hypothesized that the presence of cellular material was the result of an ICG-induced alteration of the cleavage plane from the retinal surface of the ILM to the innermost retinal layers. ${ }^{26}$ Although large retinal cellular fragments were noted after ICG staining, cellular retinal fragments in a lesser degree were also seen when ICG had not been used for staining and fragments appeared only in areas of folded ILM in specimens obtained during surgery for epimacular membranes. ${ }^{34}$ It was suggested that this observation may be related to the increased rigidity of a multilayered structure such as an epimacular membrane compared with the bare ILM, and the presence of retinal debris was interpreted as the result of a mechanical trauma during peeling. ${ }^{34}$ The presence of sub-ILM changes in terms of cellular fragments was confirmed by a recent study by Hiscott and colleagues ${ }^{35}$ suggesting that the plane of separation during ILM peeling for epiretinal membrane surgery may be altered. They concluded that this alteration was not related to the use of the dye, but should be interpreted as a result of the ERM formation or of the ERM-inducing pathology such as the modulation of GFAP within Müller cells or the continuity between components of epimacular membranes and the retina through pores of the ILM, which may increase the adhesion forces between these cells and the ILM..$^{35,36}$ In addition, one may hypothesize that an epiretinal membrane, which is peeled off along with the underlying ILM, is stiffer compared with the ILM alone as removed during macular hole surgery. However, when taking together the described cellular interactions and our observations of increased rigidity in the stained ILM, regardless which dye is used, it is quite comprehensible that there is an increased likelihood to alter the cleavage plane during vitreoretinal surgeries both for epimacular membranes and macular holes to the inner retinal layers, because the rigidity is altered either by the presence of epimacular tissue or by the use of certain vital dyes.

The present investigation has some limitations. One needs to consider that AFM provides measurements of the surface of the tissue with a limited penetration. It is known that both ICG and BB do not penetrate the ILM, the staining effect is limited to the tissue surface.

Therefore, a stiffening effect as measured by AFM might be more pronounced at the surface of the stained tissue. In addition, AFM measurements as performed herein do not represent direct measurements of the changes of the tensile strength of the ILM. Nevertheless, as the ILM represents an irregularly structured meshwork of various fibers, one may transfer our findings and conclude that 
an increase in the stiffness measured at the surface correlates to an increase in the tensile strength, which would not hold for regularly structured tissues.

Against the background of the present study, it will be interesting to evaluate potential influences of different dye concentrations and exposure times. In addition, potential effects of age or sex of the patients as well as of other underlying diseases may be evaluated in a larger sample size.

\section{Conflict of interest}

The authors declare no conflict of interest.

\section{Acknowledgements}

We thank Renate Scheler, TA, for her excellent technical support (as usual).

\section{References}

1 Brooks Jr HL. Macular hole surgery with and without internal limiting membrane peeling. Ophthalmology 2000; 107: 1939-1948.

2 Haritoglou C, Gass CA, Schaumberger M, Ehrt O, Gandorfer A, Kampik A. Macular changes after peeling of the internal limiting membrane in macular hole surgery. $A m$ J Ophthalmol 2001; 132: 363-368.

3 Eckardt C, Eckardt U, Groos S, Luciano L, Reale E. Entfernung der membrane limitans interna bei Makulalöchern. Ophthalmologe 1997; 94: 545-551.

4 Park DW, Dugel PU, Garda J, Sipperley JO, Thach A, Sneed SR et al. Macular pucker removal with and without internal limiting membrane peeling: pilot study. Ophthalmology 2003; 110: $62-64$.

5 Kadonosono K, Itoh N, Uchio E, Nakamura S, Ohno S. Staining of the internal limiting membrane in macular hole surgery. Arch Ophthalmol 2000; 118: 1116-1118.

6 Rodrigues EB, Meyer CH, Mennel S, Farah ME. Mechanisms of intravitreal toxicity of indocyanine green dye: implications for chromovitrectomy. Retina 2007; 27(7): 958-970.

7 Yam HF, Kwok AK, Chan KP, Lai TY, Chu KY, Lam DS et al. Effect of indocyanine green and illumination on gene expression in human retinal pigment epithelial cells. Invest Ophthalmol Vis Sci 2003; 44(1): 370-377.

8 Sato T, Ito M, Ishida M, Karasawa Y. Phototoxicity of indocyanine green under continuous fluorescent lamp illumination and its prevention by blocking red light on cultured Müller cells. Invest Ophthalmol Vis Sci 2010; 51(8): 4337-4345.

9 Hillenkamp J, Dydykina S, Klettner A, Treumer F, Vasold R, Bäumler $\mathrm{W}$ et al. Safety testing of indocyanine green with different surgical light sources and the protective effect of optical filters. Retina 2010; 30(10): 1685-1691.

10 Haritoglou C, Gandorfer A, Gass CA, Schaumberger M, Ulbig MW, Kampik A. Indocyanine green-assisted peeling of the internal limiting membrane in macular hole surgery affects visual outcome: a clinicopathologic correlation. Am J Ophthalmol 2002; 134: 836-841.
11 Tsuiki E, Fujikawa A, Miyamura N, Yamada K, Mishima K, Kitaoka T. Visual field defects after macular hole surgery with indocyanine green-assisted internal limiting membrane peeling. Am J Ophthalmol 2007; 143: 704-705.

12 Enaida H, Hisatomi T, Hata Y, Ueno A, Goto Y, Yamada Tet al. Brilliant blue $\mathrm{G}$ selectively stains the internal limiting membrane/brilliant blue G-assisted membrane peeling. Retina 2006; 26: 631-636.

13 Ueno A, Hisatomi T, Enaida H. Biocompatibility of brilliant blue $\mathrm{G}$ in a rat model of subretinal injection. Retina 2007; 27: 499-504.

14 Januschowski K, Mueller S, Spitzer MS, Schramm C, Doycheva D, Bartz-Schmidt KU et al. Evaluating retinal toxicity of a new heavy intraocular dye, using a model of perfused and isolated retinal cultures of bovine and human origin. Graefes Arch Clin Exp Ophthalmol 2012; 250(7): 1013-1022.

15 Remy M, Thaler S, Schumann RG, May CA, Fiedorowicz M, Schuettauf $\mathrm{F}$ et al. An in-vivo evaluation of brilliant blue $\mathrm{G}$ in animals and humans. Br J Ophthalmol 2008; 92: 1142-1147.

16 Rodrigues EB, Penha FM, Farah ME, de Paula Fiod Costa E, Maia M, Dib E et al. Preclinical investigation of the retinal biocompatibility of six novel vital dyes for chromovitrectomy. Retina 2009; 29(4): 497-510.

17 Baba T, Hagiwara A, Sato E, Arai M, Oshitari T, Yamamoto S. Comparison of vitrectomy with brilliant blue $\mathrm{G}$ or indocyanine green on retinal microstructure and function of eyes with macular hole. Ophthalmology 2012; 119(12): 2609-2615.

18 Halfter W, Dong S, Dong A, Eller AW, Nischt R. Origin and turnover of ECM proteins from the inner limiting membrane and vitreous body. Eye 2008; 22(10): 1207-1213.

19 Sneddon IN. The relation between load and penetration in the axisymmetric boussinesq problem for a punch of arbitrary profile. Int J Engng Sci 1965; 3: 47-57.

20 Henrich PB, Monnier CA, Halfter W, Haritoglou C, Strauss RW, Lim RY et al. Nanoscale topographic and biomechanical studies of the human internal limiting membrane. Invest Ophthalmol Vis Sci 2012; 53(6): 2561-2570.

21 Heegaard S, Jensen OA, Prause JU. Structure and composition of the inner limiting membrane of the retina. SEM on frozen resin-cracked and enzyme-digested retinas of Macaca mulatta. Graefes Arch Clin Exp Ophthalmol 1986; 224: $355-360$.

22 Sebag J. Anatomy and pathology of the vitreo-retinal interface. Eye 1992; 6: 541-552.

23 Streeten BA. Disorders of the vitreous. In: Garner A, Klintworth GK (eds) Pathophysiology of Ocular Disease-A Dynamic Approach, part B. Marcel Dekker: New York, 1982; 1381-1419.

24 Candiello J, Balasubramani M, Schreiber EM, Cole GJ, Mayer U, Halfter $\mathrm{W}$ et al. Biomechanical properties of native basement membranes. FEBS J 2007; 274(11): 2897-2908.

25 Halfter W, Candiello J, Hu H, Zhang P, Schreiber E, Balasubramani M. Protein composition and biomechanical properties of in vivo-derived basement membranes. Cell Adh Migr 2012; 7(1): 64-71.

26 Gandorfer A, Haritoglou C, Gandorfer A, Kampik A. Retinal damage from indocyanine green in experimental macular surgery. Invest Ophthalmol Vis Sci 2003; 44: 316-323.

27 Wollensak G. Biomechanical changes of the internal limiting membrane after indocyanine green staining. Dev Ophthalmol 2008; 42: 82-90. 
28 Wollensak G, Spoerl E, Wirbelauer C, Pham DT. Influence of indocyanine green staining on the biomechanical strength of porcine internal limiting membrane. Ophthalmologica 2004; 218(4): 278-282.

29 Foote CS. Definition of type I and type II photosensitized oxidation. Photochem Photobiol 1991; 54: 659.

30 Andley U. Photooxidative stress. in Albert DM, Jakobiec F (eds) Principles and Practice of Ophthalmology. WB Saunders Co: Philadelphia, 1992; vol 1, pp 575-590.

31 Engelbrecht NE, Freeman J, Sternberg Jr P, Aaberg Jr TM, Aaberg Jr TM, Martin DF et al. Retinal pigment epithelial changes after macular hole surgery with indocyanine greenassisted internal limiting membrane peeling. Am J Ophthalmol 2002; 133: 89-94.

32 Uemura A, Kanda S, Sakamoto Y, Kita H. Visual field defects after uneventful vitrectomy for epiretinal membrane with indocyanine green-assisted internal limiting membrane peeling. Am J Ophthalmol 2003; 136: 252-257.

33 Engel E, Schraml R, Maisch T, Kobuch K, König B, Szeimies $\mathrm{RM}$ et al. Light-induced decomposition of indocyanine green. Invest Ophthalmol Vis Sci 2008; 49(5): 1777-1783.

34 Haritoglou C, Gandorfer A, Gass CA, Schaumberger M, Ulbig MW, Kampik A. The effect of indocyanine-green on functional outcome of macular pucker surgery. Am J Ophthalmol 2003; 135: 328-337.

35 Kenawy N, Wong D, Stappler T, Romano MR, Das RA, Hebbar $\mathrm{G}$ et al. Does the presence of an epiretinal membrane alter the cleavage plane during internal limiting membrane peeling? Ophthalmology 2010; 117(2): 320-323

36 Gandorfer A, Schumann R, Scheler R, Haritoglou C, Kampik A. Pores of the inner limiting membrane in flatmounted surgical specimens. Retina 2011; 31(5): 977-981. 